\title{
Survival of Patients with Primary Cardiac Tumors in an 11-Year Single Center Study
}

\author{
Aizezi Maimaitiaili, Wei-min Zhang, Yong-zhong Guo, Doshahar Elmaik, Nian-he Tang, Zong-gang Zhang \\ Department of Cardiac Surgery, People's Hospital of Xinjiang Uygur Autonomous Region, Urumqi, China
}

\section{ABSTRACT}

Background: Primary cardiac tumors are rare, but few studies have examined the relationship between risk factors and the prognosis. The aim of this study was to provide a survival analysis and risk factors for mortality in patients with primary cardiac tumors.

Methods: We retrospectively enrolled 71 patients diagnosed with primary cardiac tumors from June 2006 to November 2017 in our hospital. Patients' population characteristics, treatment information, pathology, and follow-up data were obtained and analyzed.

Results: Of the 71 patients, 60 cases were benign, and 11 cases were malignant. Sex, age, New York Heart Association classification, the percentage of peripheral embolism, and surgery had no significant difference between benign and malignant groups $(P>.05)$, but the percentage of arrhythmia, leg edema, and mortality rate was higher in the malignant tumor group than in the benign tumor group $(P<.05)$. Compared with the benign tumor group, the percentage of biatrial lesions in the malignant tumor group was significantly higher $(P<.05)$. Moreover, Independent risk factors included the treatment choice, pathology type, and number of tumor lesions $(P<.05)$.

Conclusion: Our study suggests that conservative therapy, malignant cardiac tumor, and biatrial tumor lesion are independent risk factors for poor prognosis.

\section{]INTRODUCTION}

Primary cardiac tumors are extremely rare, with an autopsy incidence ranging from $0.001 \%$ to $0.030 \%$. Almost $75 \%$ of cardiac tumors are benign, with myxomas the most frequent type; and nearly $25 \%$ of cardiac tumors are malignant, with sarcomas predominate [Butany 2005]. Of note, recent data from 18 cancer registries in the United States found that the incidence of primary malignant cardiac tumors was 34 cases per 100 million and increased obviously from 1973 to 2011 [Oliveira 2015]. As soon as the diagnosis with cardiac tumor is confirmed, the patient should be

Received October 29, 2018; received in revised form December 3, 2018; accepted fanuary 2, 2019.

Correspondence: Zong-gang Zhang, MD, Department of Cardiac Surgery, People's Hospital of Xinjiang Uygur Autonomous Region, 91 Tianchi Road, Urumqi City 830001, Xinjiang province, China; +86-(0)991-13999173762; fax: +86-(0)991-8565302 (e-mail: zhangzg196219@163.com). treated with surgical resection. Although surgical treatment is considered as a safe and highly effective strategy associated with good early and long-term outcomes [Bakaeen 2003; Yu 2014; Li 2017; Mkalaluh 2017; Saad 2018], a few literature reports revealed that some risk factors may affect the prognosis for patients with primary cardiac tumors, such as refusal of surgery [Yu 2014], operation method [Li 2017], pathology type [Bakaeen 2003; Simpson 2008; Oliveira 2015; Mkalaluh 2017; Nomoto 2017], and New York Heart Association (NYHA) classification [Bakaeen 2003]. Conversely, age, sex, and race did not have a significant impact on survival rate [Saad 2018].

Considering the lack of data in the literature on the risk factors for prognosis of primary cardiac tumors, we retrospectively analyzed 71 patients in our single center over the past 11 years, and therefore sought to provide an in-depth analysis of survival and risk factors for mortality in patients with primary cardiac tumors.

\section{MATERIALS AND METHODS}

\section{Study Population}

We conducted a retrospective analysis of all cardiac tumors in the patient database of the People's Hospital of Xinjiang Uygur Autonomous Region from June 2006 to November 2017. The patients who had metastatic disease at the time of diagnosis were excluded. Finally, 71 consecutive patients with primary cardiac tumors were screened, including 60 cases of benign tumor and 11 cases of malignant tumor. This study protocol was approved by the Institutional Ethics Committee of People's Hospital of Xinjiang Uygur Autonomous Region.

\section{Data Recording}

Patient information was obtained from hospital records, including age, sex, NYHA classification, symptom, operation, and tumor characteristics. Pathological diagnosis of each patient who had received surgery was obtained from our hospital's pathology division, and the remaining patients who refused surgery were diagnosed by using echocardiography and magnetic resonance. Furthermore, long-term follow-up data were collected at outpatient clinics or in telephone interviews for all participants. Follow-up time was calculated either to death or to the last contact with the living patient, and surviving patients were censored at the last follow-up date. Median follow-up was 48.0 months (range, 3-120 months). 
Table 1. Baseline Patient Characteristics

\begin{tabular}{|c|c|c|c|}
\hline Variable & Benign $(n=60)$ & Malignant $(n=11)$ & $P$ \\
\hline Sex & & & .255 \\
\hline Male & 27 & 7 & \\
\hline Female & 33 & 4 & \\
\hline Age, years (mean $\pm S D)$ & $49.15 \pm 13.05$ & $41.09 \pm 17.86$ & .088 \\
\hline NYHA classification* & & & .680 \\
\hline I and II & 50 & 8 & \\
\hline III and IV & 10 & 3 & \\
\hline Arrhythmia & & & .011 \\
\hline Yes & 6 & 5 & \\
\hline No & 54 & 6 & \\
\hline Leg edema & & & .025 \\
\hline Yes & 12 & 6 & \\
\hline No & 48 & 5 & \\
\hline Peripheral embolism & & & 1.000 \\
\hline Yes & 4 & 0 & \\
\hline No & 56 & 11 & \\
\hline Surgery & & & .169 \\
\hline Yes & 57 & 9 & \\
\hline No & 3 & 2 & \\
\hline Vital status & & & $<.001$ \\
\hline Alive & 59 & 6 & \\
\hline Dead & 1 & 5 & \\
\hline
\end{tabular}

*NYHA, New York Heart Association.

\section{Statistical Analysis}

All statistical analyses were performed using IBM SPSS Statistics for Windows, Version 19.0 (IBM Corp., Armonk, NY, USA). Continuous data were presented as mean \pm standard deviation, and the Kolmogorov-Smirnov test was used to check for normal distribution before any additional analysis. Categorical data were described as number and percentage. Differences between benign and malignant cardiac tumors were compared by using the chi-square test or the Fisher exact test, and by using independent $t$ test for continuous variables. Longterm survival was calculated and graphically presented by using the Kaplan-Meier method; both the log-rank and Breslow tests were used for all survival differences. The variables which were significantly $(P<.05)$ associated with the survival rate were analyzed by the multivariate logistic regression analysis to identify the independent factors. All reported $P$ values are 2 -sided, and $P$ values less than .05 and .01 were considered statistically significant and highly statistically significant, respectively.

\section{Trial Registration}

This is a retrospective study, which was not registered in any trial registry.
Table 2. Characteristics of the Cardiac Tumors

\begin{tabular}{lccc}
\hline Variable & Benign $(\mathrm{n}=60)$ & Malignant $(\mathrm{n}=11)$ & $P$ \\
\hline $\begin{array}{l}\text { Number of tumor lesions } \\
\text { Solitary lesion }\end{array}$ & 57 & 8 & .044 \\
$\quad$ Biatrial lesion & 3 & 3 & $<.001$ \\
Tumor lesion location & 55 & & \\
Left atrium & 6 & 2 & \\
Right atrium & 0 & 2 & \\
Left ventricle & 2 & 1 & \\
Right ventricle & 0 & 5 & \\
Pericardium & & - \\
Pathology & 54 & - \\
Myxoma & 3 & 8 \\
Fibroelastoma & 3 & 3 \\
Lipoma & - & \\
Sarcoma & - & \\
Mesothelioma & & \\
& & & \\
RESULTS & & & \\
& & &
\end{tabular}

\section{Patient Characteristics}

In this study, 71 patients met our inclusion criteria, and the baseline characteristics are described in Table 1. The result showed that $84.51 \%$ of the primary cardiac tumors were benign $(n=60)$ and $15.49 \%$ of the primary cardiac tumors were malignant $(n=11)$. Both sex and age had no significant difference between benign and malignant groups $(P>.05)$. According to NYHA classification grading of cardiac failure, benign and malignant tumors had no statistical significance between NYHA I and II $(83.33 \%$ versus $72.73 \%)$ and between NYHA III and IV $(16.67 \%$ versus $27.27 \%$ ).

Patients with cardiac tumors presented diverse symptoms: 11 patients $(15.49 \%)$ had arrhythmia including atrial fibrillation $(n=5)$, ventricular tachycardia $(n=3)$, third-degree atrioventricular block $(\mathrm{n}=3)$; 18 patients $(25.35 \%)$ had leg edema; and 4 patients $(5.63 \%$ ) had peripheral thromboembolism that only happened in benign tumors. Of note, the percentage of arrhythmia (45.45\% versus $10.00 \%, P=.011)$ and of leg edema $(54.55 \%$ versus $20.00 \%, P=.025)$ in patients with malignant tumors were significantly higher than in those with benign tumors.

In the treatment, 66 patients underwent surgery, but 3 cases of benign tumor and 2 cases of malignant tumor chose conservative treatment, and the 2 malignant tumor cases who refused surgery died. At 11-year follow-up, only 1 patient from the benign tumor group died because of cardiac arrest, and 5 patients from the malignant tumor group died because of cardiac arrest $(n=2)$, malignant arrhythmia $(n=2)$, and cardiogenic shock $(\mathrm{n}=1)$. 
Table 3. Multivariate Logistic Regression Analysis Model for Mortality of Cardiac Tumors*

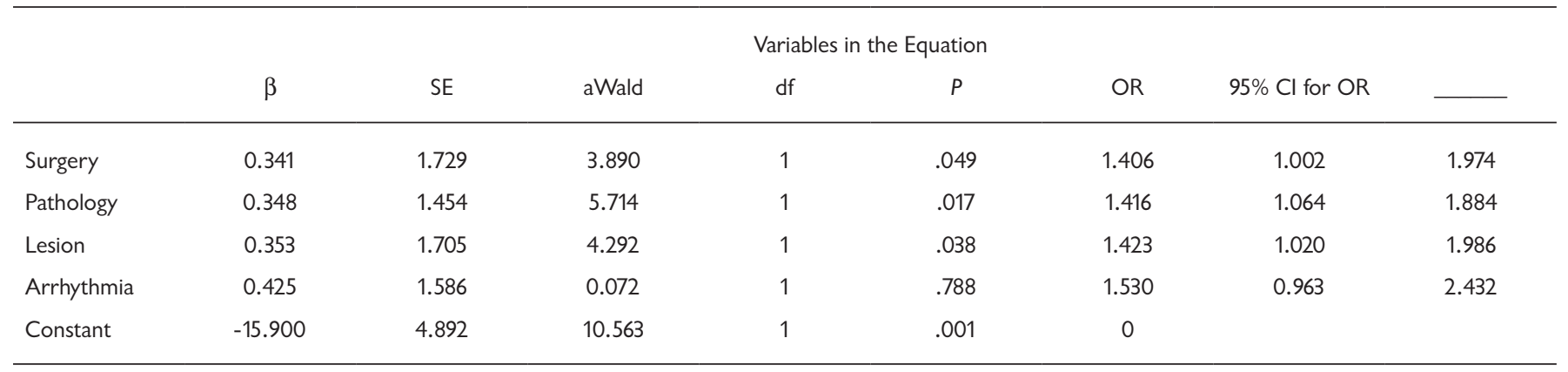

*SE, standard error; Wald, chi-square value; $\mathrm{Cl}$, confidence interval.
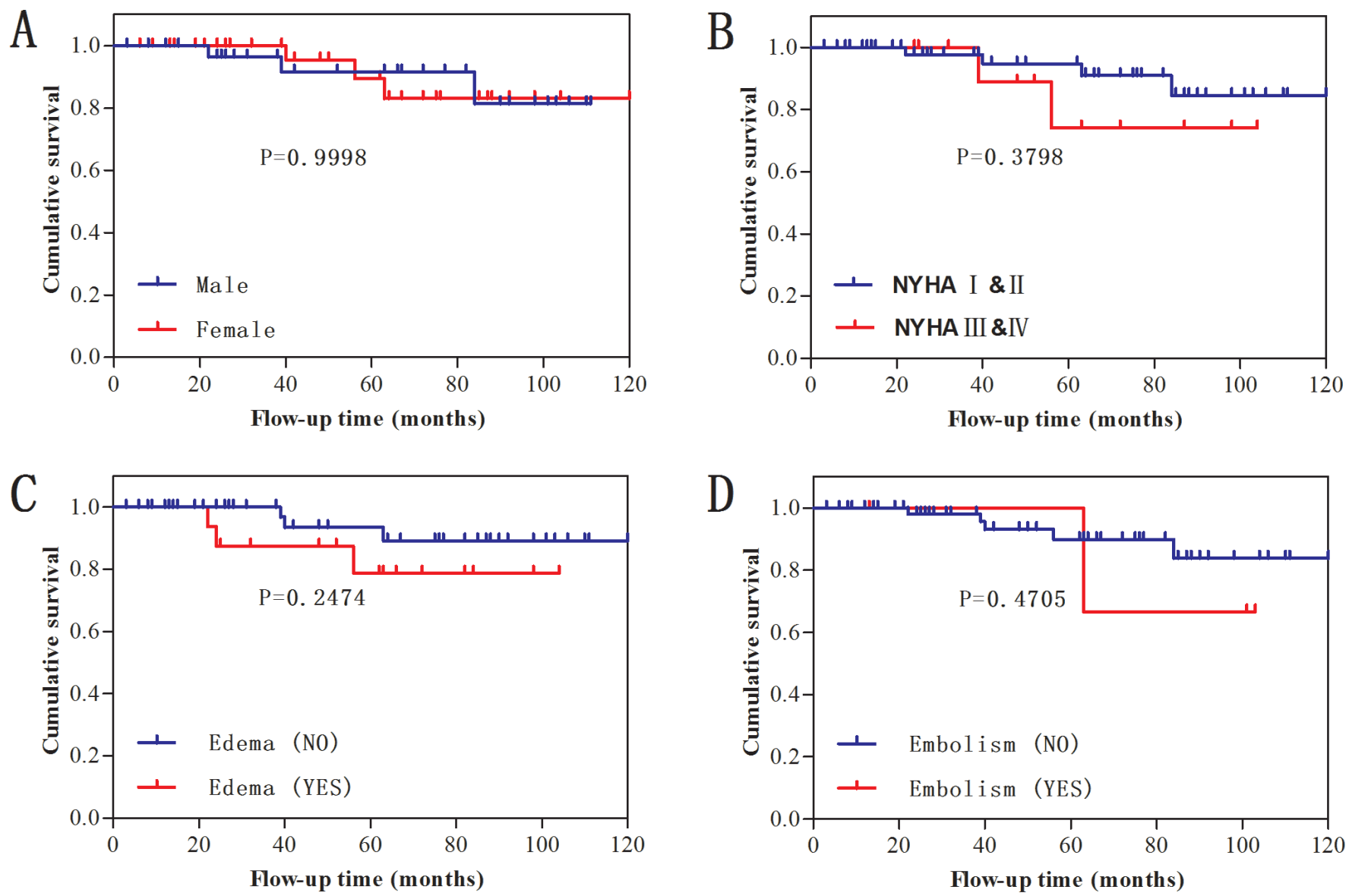

Figure 1. Comparative survival of primary cardiac tumors by sex (A), NYHA grade (B), edema (C), and embolism (D).

\section{Tumor Characteristics}

Table 2 shows tumor location and pathology of cardiac tumors in detail. A total of 77 lesions were visualized by using echocardiography and magnetic resonance in 71 patients. Tumors that occurred in 2 chambers were seen in 6 patients, including 3 cases in the left atrium and right atrium, 2 cases in the right atrium and right ventricle, 1 case in the left atrium and left ventricle, whereas 65 patients had a tumor in a single chamber. Compared with benign tumors, the percentage of biatrial lesions $(27.27 \%$ versus $5.0 \%)$ in malignant tumors was significantly higher $(P=.044)$. In cases of benign tumors, the left atrium was the most frequent location $(\mathrm{n}=55,87.30 \%)$, whereas malignant tumors occurred in any chamber of the heart, but are frequently found in the pericardium $(\mathrm{n}=5,45.45 \%)$ and right atrium $(\mathrm{n}=4,36.36 \%)$. The most common benign tumor was myxoma $(n=54,90.00 \%)$, 
A
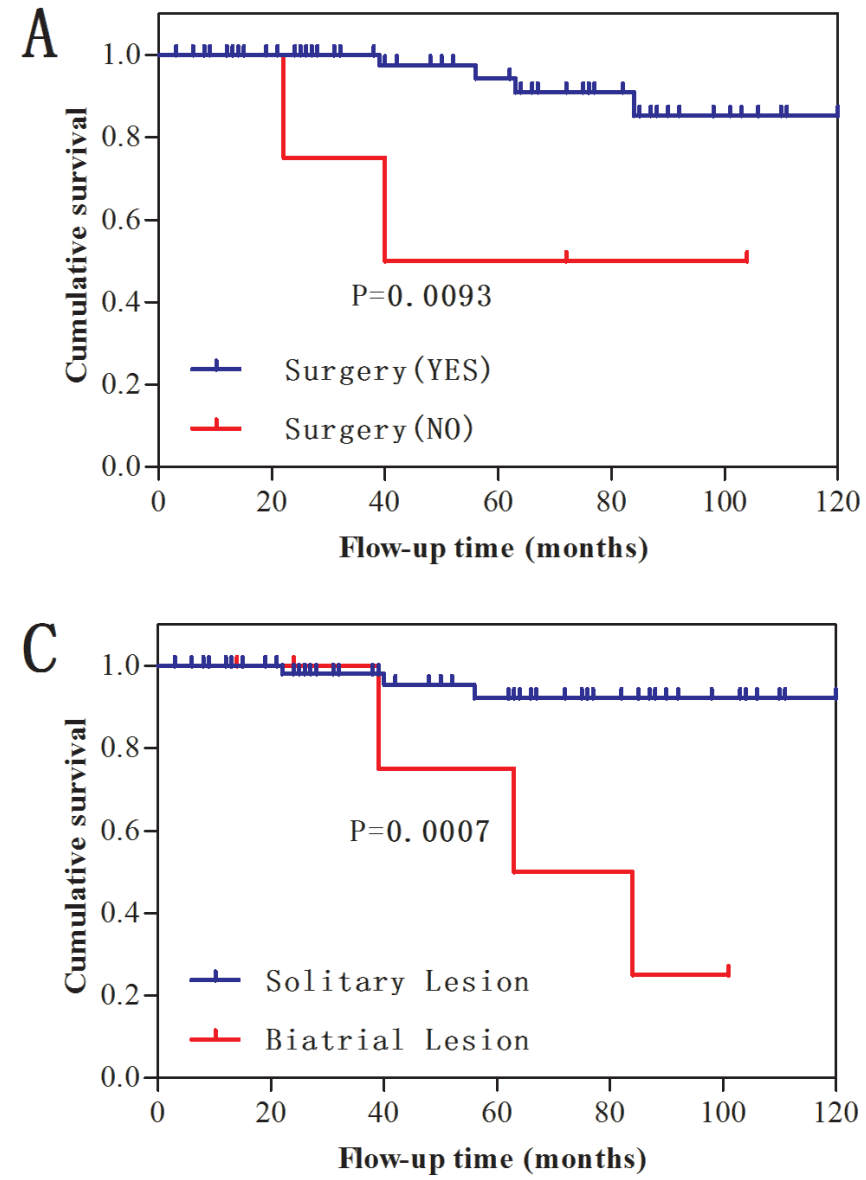

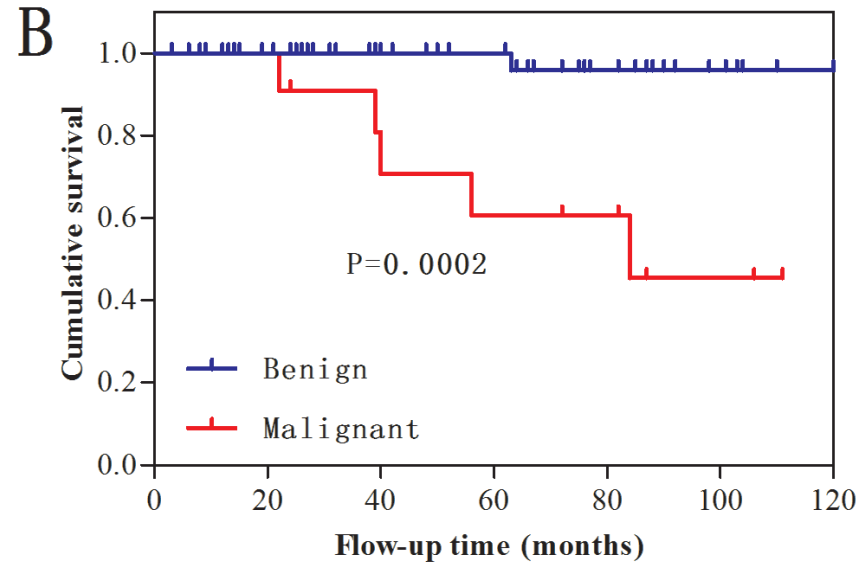

D

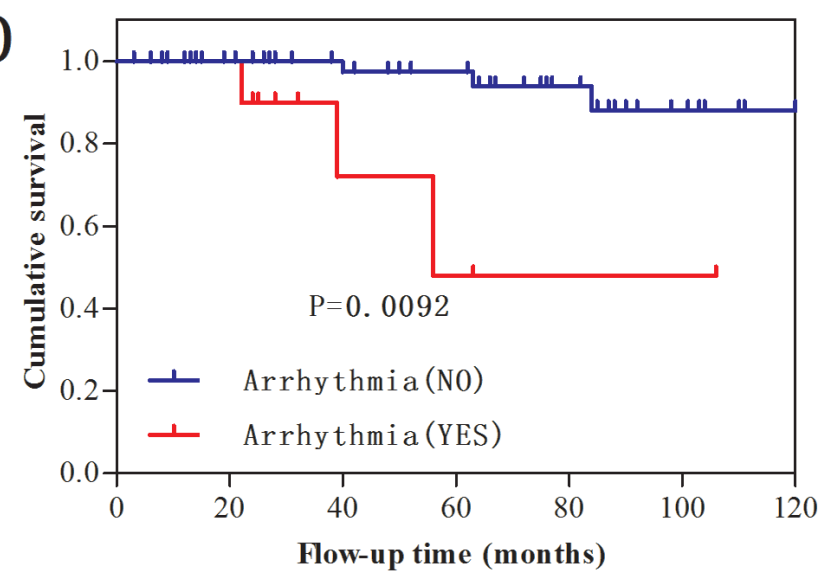

Figure 2. Comparative survival of primary cardiac tumors by surgery (A), pathology type (B), number of tumor lesions (C), and arrhythmia (D).

followed by fibroelastoma $(\mathrm{n}=3,5.00 \%)$ and lipoma $(\mathrm{n}=3$, $5.00 \%)$. Moreover, cardiac sarcomas $(\mathrm{n}=8,72.73 \%)$ formed the largest group of malignant tumors, followed by mesothelioma $(\mathrm{n}=3,27.27 \%)$.

\section{Mortality}

At the last follow up of 11 years, malignant tumors had a higher mortality rate than benign tumors (45.45\% versus $1.67 \%, P<.001)$. The survival curves showed that sex, NYHA classification, leg edema, and peripheral embolism had no statistically significant impact on survival (Figures 1A-1D). However, surgical treatment was associated with an improvement in the overall survival (logrank $P=.009$, Breslow $P=.001$, Figure $2 \mathrm{~A}$ ), and patients with malignant cardiac tumors had significantly lower survival rate than those with benign tumors (log-rank $P=.000$, Breslow $P=.000$, Figure 2B). Additionally, patients with biatrial lesions (log-rank $P=.001$, Breslow $P=.009$, Figure 2C) and arrhythmia (log-rank $P=.001$, Breslow $P=.001$, Figure 2D) had a significantly lower survival rate. As shown in Table 3 , the treatment choice, pathology type, and number of tumor lesions were the independent risk factors for mortality confirmed by the multivariate logistic regression analysis $(P<.05)$.

\section{DISCUSSION}

In this study, we presented the patient characteristics, tumor lesions, and mortality of patients with primary cardiac tumors in our center. Compared with most of the previous reports, our research uniquely collected data on both benign and malignant cardiac tumors to provide a survival analysis and risk factors for prognosis.

In our data analysis, $84.51 \%$ of all the primary cardiac tumors were benign, which was similar to previous studies in Spain (84.90\%) [Barreiro 2013], China (79.47\%) [Yin 2016], and Germany (77.00\%) [Hoffmeier 2014]. In contrast, the remaining cardiac tumors are rare, and we found that a percentage of $15.49 \%$ of cardiac tumors were malignant and that various types of sarcoma together formed $72.73 \%$ of all malignant cardiac tumors, followed by mesothelioma. The percentage of malignant cardiac tumors in our study was consistent with other reports [Habertheuer 2015; Yin 2016; Mkalaluh 2017]. However, malignant cardiac tumors were frequently found in the right atrium [Hoffmeier 2014], but our results showed that the pericardium was the most often location, whereas the right atrium came in as the second. Interestingly, we also reported that the percentage of biatrial lesions in malignant tumors was significantly higher than in benign tumors. 
Patients with cardiac tumors often have diverse clinical presentations depending on the size and site of the tumor, regardless of tumor type [Roberts 1997]. According to the experience of $\mathrm{Li}$ et al, about $18.7 \%$ of patients were asymptomatic [Li 2017]. Although symptoms such as dyspnea, angina, embolism, and arrhythmia are more frequently seen in cardiac tumors [Barnes 2014; Li 2017; Mkalaluh 2017], we found that the main clinical presentations were arrhythmia, leg edema, and peripheral embolism. However, $5.63 \%$ of patients had peripheral embolism in our center; this was lower than the reports of Dias et al (19.80\%) [Dias 2014] and Strecker et al (15.50\%) [Strecker 2012]. Particularly, the events of peripheral embolism mostly happen in myxomas of benign tumors. As the reasons for it, Butany et al suggested that myxomas tend to cause embolisms because of their gelatinous structure [Butany 2005].

Since the first excision of intra-atrial myxoma was performed by Clarence Crafoord in 1954 [Chitwood 1992], cardiac tumors' resection gradually became a common, safe, and highly effective strategy associated with good early and long-term outcomes[Mkalaluh 2017; Li 2017; Kośmider 2013]. The rate of operation was $92.96 \%$ in our institution for both benign and malignant cardiac tumors. The overall cumulative survival after cardiac tumor excision at 30 days, 1 year, 5 years, and 15 years was $94 \%, 85 \%$, $72 \%$, and $59 \%$, respectively [Mkalaluh 2017]. Another report also showed that the 5-year survival rates were $83 \%$ for benign tumors, $30 \%$ for malignant tumors, and $26 \%$ for cardiac metastases [Hoffmeier 2014]. Excellent early and late surgical results can be obtained in patients with benign tumors. However, operation therapy of malignant cardiac tumors, which are difficult to excise completely, continues to have a poor prognosis [Bossert 2005; Barreiro 2013; Kośmider 2013; Yin 2016]. Indeed, Yu et al revealed that there were no differences in survival between refusal of surgery and surgery with positive surgical margins and that patients with negative surgical margins had a longer survival duration than patients who had positive surgical margins [Yu 2014]. In our study, we found that patients who refused surgery had a lower survival compared with operation receivers, and that such refusal was an independent risk factor for poor prognosis, which was consistent with a previous report [Yu 2014].

In addition, we also confirmed that malignant cardiac tumors and biatrial tumor lesions were independent risk factors for lower survival rate. Many reports have demonstrated that patients with malignant cardiac tumors had worse survival, especially angiosarcomas [Mkalaluh 2017; Nomoto 2017; Simpson 2008; Bakaeen 2003; Oliveira 2015]. To our knowledge, this is the first report of the association between biatrial tumor lesions and prognosis of cardiac tumors. Moreover, sex, NYHA grade, leg edema, and peripheral embolism had no effect on survival in our study, which was consistent with previous report [Saad 2018]. Conversely, Bakaeen et al reported that patients with NYHA III and IV had worse survival than those with NYHA I and II [Bakaeen 2003].

\section{CONCLUSIONS}

Primary cardiac tumors are rare and some risk factors may affect the prognosis. Our study suggests that conservative therapy, malignant cardiac tumors, and biatrial tumor lesions are independent risk factors for poor prognosis.

\section{REFERENCES}

Bakaeen FG, Reardon MJ, Coselli JS, et al. 2003. Surgical outcome in 85 patients with primary cardiac tumors. Am J Surg 186(6):641-7; discussion 647.

Barnes H, Conaglen P, Russell P, Newcomb A. 2014. Clinicopathological and surgical experience with primary cardiac tumors. Asian Cardiovasc Thorac Ann 22(9):1054-8.

Barreiro M, Renilla A, Jimenez JM, et al. 2013. Primary cardiac tumors: 32 years of experience from a Spanish tertiary surgical center. Cardiovasc Pathol 22(6):424-7.

Bossert T, Gummert JF, Battellini R, et al. 2005. Surgical experience with 77 primary cardiac tumors. Interact Cardiovasc Thorac Surg 4(4):311-5.

Butany J, Nair V, Naseemuddin A, Nair GM, Catton C, Yau T. 2005. Cardiac tumours: diagnosis and management. Lancet Oncol 6(4):219-28.

Chitwood WR Jr. 1992. Clarence Crafoord and the first successful resection of a cardiac myxoma. Ann Thorac Surg 54(5):997-8.

Dias RR, Fernandes F, Ramires FJA, Mady C, Albuquerque CP, Jatene FB. 2014. Mortality and embolic potential of cardiac tumors $=$ Mortalidade e potencial embólico dos tumores cardíacos. Arq Bras Cardiol 103(1):13-8. English, Portuguese.

Habertheuer A, Laufer G, Wiedemann D, et al. 2015. Primary cardiac tumors on the verge of oblivion: a European experience over 15 years. J Cardiothorac Surg 10:56.

Hoffmeier A, Sindermann JR, Scheld HH, Martens S. 2014. Cardiac tumors--diagnosis and surgical treatment. Dtsch Arztebl Int 111(12):205-11.

Kośmider A, Jaszewski R, Marcinkiewicz A, Bartczak K, Knopik J, Ostrowski S. 2013. 23-year experience on diagnosis and surgical treatment of benign and malignant cardiac tumors. Arch Med Sci 9(5):826-30.

Li S, Gao C. 2017. Surgical experience of primary cardiac tumor: singleinstitution 23-year report. Med Sci Monit 23:2111-7.

Mkalaluh S, Szczechowicz M, Torabi S, et al. 2017. Surgical treatment of cardiac tumors: insights from an 18-year single-center analysis. Med Sci Monit 23:6201-9.

Nomoto N, Tani T, Konda T, et al. 2017. Primary and metastatic cardiac tumors: echocardiographic diagnosis, treatment and prognosis in a 15-years single center study. J Cardiothorac Surg 12(1):103.

Oliveira GH, Al-Kindi SG, Hoimes C, Park SJ. 2015. Characteristics and survival of malignant cardiac tumors: a 40 -year analysis of $>500$ patients. Circulation 132(25):2395-402.

Roberts WC. 1997. Primary and secondary neoplasms of the heart. Am J Cardiol 80(5): 671-82.

Saad AM, Abushouk AI, Al-Husseini MJ, et al. 2018. Characteristics, survival and incidence rates and trends of primary cardiac malignancies in the United States. Cardiovasc Pathol 33:27-31.

Simpson L, Kumar SK, Okuno SH, et al. 2008. Malignant primary cardiac tumors: review of a single institution experience. Cancer 112(11):2440-6. 
Strecker T, Rösch J, Weyand M, Agaimy A. 2012. Primary and metastatic cardiac tumors: imaging characteristics, surgical treatment, and histopathological spectrum: a 10-year-experience at a German heart center. Cardiovasc Pathol 21(5):436-43.
Yin L, He D, Shen H, et al. 2016. Surgical treatment of cardiac tumors: a 5 -year experience from a single cardiac center. J Thorac Dis 8(5):911-9.

Yu L, Gu T, Shi E, et al. 2014. Primary malignant cardiac tumors. J Cancer Res Clin Oncol 140(6):1047-55. 